\title{
Insatisfação corporal em adolescentes brasileiros de municípios de pequeno porte de Minas Gerais
}

\author{
Body dissatisfaction in Brazilian adolescents from \\ small municipalities of Minas Gerais \\ Valter Paulo Neves Miranda', Maria Aparecida Conti ${ }^{2}$ Ronaldo Bastos ${ }^{3}$, Maria Elisa Caputo Ferreira'
}

\section{Palavras-chave \\ Imagem corporal, adolescente, sexo, estado nutricional.}

\section{RESUMO}

Objetivo: Analisar a prevalência de insatisfação corporal em adolescentes e sua relação com idade, sexo e estado nutricional. Métodos: A amostra foi composta por escolares do Ensino Médio de municípios com até cinco mil habitantes de Minas Gerais. O Body Shape Questionnaire (BSQ) e a Escala Evaluacion de Insatisfacion Corporal para Adolescentes (EEICA) avaliaram a insatisfação corporal. A idade, o sexo, o peso e a estatura, para o cálculo do índice de massa corporal, foram coletados nas escolas. Para análises estatísticas foram utilizados testes de variância e análise de correspondência. Resultados: Dos 413 participantes, 49,2\% e 50,8\% estavam no período intermediário e final da adolescência, respectivamente. Desse total, 178 eram meninos e 235 eram meninas e a maioria (71,9\%), eutrófico. A média do BSQ foi de $66,78 \pm 29,63$ pontos, sendo que $26,4 \%$ apresentaram algum nível de insatisfação corporal e, pela EEICA, a média de insatisfação foi de 17,96 $\pm 11,7$ pontos. As análises de correspondência simples e múltipla indicaram o sexo e o estado nutricional como modalidades com maiores diferenças de insatisfação corporal. Conclusão: Apesar de baixa prevalência de insatisfação corporal, alguns adolescentes apresentaram grave insatisfação com sua imagem corporal, principalmente as meninas e adolescentes com sobrepeso ou obesidade.

\section{ABSTRACT}

Objective. To analyze the prevalence of body dissatisfaction and its correlations with age, sex and the nutritional status of teenagers. Methods: The sample consisted of high school students in cities with up to five thousand inhabitants in the state of Minas Gerais, Brazil. The Body Shape Questionnaire (BSQ) and the Escala Evaluacion de Insatisfacion Corporal para Adolescentes (EElCA) were instruments used to assess the adolescents' body dissatisfaction. The age, sex, weight and height to calculate body mass index was also collected in schools. For statistical analyses the variance test and correspondence analysis were used. Results: Out of 413 participants, 49.2\% and $50.8 \%$ were in the intermediate and final phases of adolescence, respectively. Of this total, 178 were boys and 235 were girls and, most (71.9\%) eutrophic. The BSQ score mean was $66.78 \pm$ 29.63 and $26.4 \%$ had some level of body dissatisfaction; the EEICA average score for body dissatisfaction was $17.96 \pm 11.7$. The single and multiple correspondence analyses have shown that

1 Universidade Federal de Juiz de Fora (UFJF), Faculdade de Educação Física e Desportos, Grupo de Estudos Corpo e Diversidade Humana. 2 Universidade de São Paulo (USP), Faculdade de Medicina, Hospital das Clínicas, Instituto de Psiquiatria, Ambulatório de Bulimia e Transtornos Alimentares.

3 Universidade Federal de Juiz de Fora (UFJF), Instituto de Ciências Exatas, Departamento de Estatística.

Endereço para correspondência: Valter Paulo Neves Miranda

Rua Dr. Gil Horta, 163, ap. 402 - Centro - 36016-400 - Juiz de Fora, MG

Tel.: (32) 9928-9878

E-mail: vpnmiranda@yahoo.com.br 


\section{Keywords}

Body image, adolescent, sex, nutritional status. sex and nutritional status are modalities with higher differences in rates of body dissatisfaction. Conclusion: In spite of the low prevalence of body dissatisfaction, some teenagers from the small towns surveyed had severe dissatisfaction with their body image, especially girls and adolescents with overweight and suffering from obesity.

\section{INTRODUÇÃO}

A insatisfação corporal pode ser compreendida como um incômodo que o indivíduo vivencia em relação aos aspectos de sua aparência física'. Nos últimos 20 anos, pesquisadores intensificaram suas investigações, observando que esse sentimento negativo com a imagem corporal pode ser mais evidente durante a adolescência devido às transformações sofridas na puberdade, em decorrência dos padrões de beleza ofertados pela mídia, com a valorização de formas físicas supostamente ideais².

A adolescência é uma fase de transição no desenvolvimento entre a infância e a idade adulta, envolvendo grandes mudanças físicas, cognitivas e psicossociais relacionadas entre $\mathrm{si}^{3}$. Durante a adolescência, pode ocorrer grande variação do estado nutricional em um período muito curto da vida do ser humano. O aumento do peso pode ser considerado um dos principais fatores relacionados à alta prevalência de insatisfação corporal, principalmente entre as meninas ${ }^{4,5}$. Algumas pesquisas realizadas no Brasil mostram que a insatisfação com a imagem corporal durante essa fase da vida pode atingir de $40 \%$ a $60 \%$ em ambos os sexos ${ }^{6,7}$, além de ser um fator que pode evoluir o desenvolvimento dos transtornos alimentares ${ }^{8}$.

A percepção corporal, as atitudes, as crenças, as práticas, as representações, os sentimentos, as sensações e os comportamentos relativos ao corpo são facetas que envolvem o fenômeno da imagem corporal'. Para avaliar esses aspectos, o pesquisador lançará mão de instrumentos validados e adaptados transculturalmente para a população que está sendo investigada. Os questionários e escalas autoaplicáveis são instrumentos mais adequados à avaliação dos aspectos que compõem a imagem corporal, quer em estudos epidemiológicos, como também em amostras clínicas.

A literatura aponta que o adolescente pode sentir-se mais insatisfeito com sua aparência física por ser influenciado e pressionado pelas tendências sociais e culturais, e, assim, materializar, no próprio corpo, o ideal de forma física pertencente à cultura ao qual está inserido ${ }^{10}$. São carentes as pesquisas que abordam a insatisfação corporal de jovens moradores de diferentes regiões sociodemográficas ${ }^{6,7}$, além de não levar em consideração alguns fatores associados, como: idade, sexo e estado nutricional. Visto isso este estudo epidemiológico pretendeu analisar a prevalência de insatisfação corporal de adolescentes domiciliados em cidades pequenas.

\section{MÉTODOS}

O estudo realizado foi do tipo epidemiológico transversal de base escolar, desenvolvido pelo Laboratório de Estudos do Corpo (LABESC) da Faculdade de Educação Física e Desportos (FAEFID) da Universidade Federal de Juiz de Fora (UFJF).

\section{POPULAÇÃO E AMOSTRA}

A população foi constituída por adolescentes, de 15 a 19 anos $^{11}$, de ambos os sexos, regularmente matriculados nas escolas da rede pública de municípios de pequeno porte ${ }^{12}$ do interior da Zona da Mata mineira, com até 5.000 habitantes. A escolha de cidades com essas características se justifica pelo fato de serem sociodemograficamente semelhantes, principalmente em relação à sua concentração populacional (Tabela 1).

Foram observados nove municípios com as características demográficas descritas no site do Instituto Brasileiro de Geografia e Estatística (IBGE) ${ }^{13}$. Em seguida, no site da Secretaria Estadual de Educação de Minas Gerais (SEE/MG), buscaram-se informações sobre as escolas da rede pública que ofereciam ensino médio, sendo encontrada uma escola em cada cidade. A partir daí, realizou-se uma amostra por conglomerados, tendo como universo as 9 escolas (conglomerados) e uma população finita de 1.015 alunos, divididos por série e sexo.

Adotando-se uma precisão absoluta de 5 pontos para mais ou para menos na variável resposta de referência, o escore do Body Shape Questionnaire (BSQ) ${ }^{14}$, e a maior variabilidade de pontuação verificada para o sexo feminino ( $\pm 34,3$ pontos), como a pior situação ao nível de significância de $5 \%$, obteve-se um tamanho de amostra mínimo necessário igual a 129 ( $n=129)$, já feita a correção para populações finitas. Esse valor foi dividido pela menor média de sujeitos observada, ou seja, na terceira série do ensino médio $(31,33)$, dando um valor aproximadamente igual a 4 , sendo esse o número de municípios selecionados por amostragem aleatória simples. Para realizar inferências independentes por série, considerando cada uma como estrato, o tamanho amostral inicial obtido foi multiplicado por 3 (referente às três séries do ensino médio), dando um tamanho amostral final igual a 387 alunos. 
Tabela 1. Distribuição de alunos segundo séries e sexo das cidades de pequeno porte da Zona da Mata mineira, 2009

\begin{tabular}{|c|c|c|c|c|c|c|c|}
\hline \multirow{2}{*}{ Cidades } & \multicolumn{2}{|c|}{$1^{\circ} \mathrm{EM}$} & \multicolumn{2}{|c|}{$2^{\circ} \mathrm{EM}$} & \multicolumn{2}{|c|}{$3^{\circ} \mathrm{EM}$} & \multirow{2}{*}{$\begin{array}{c}\text { Total } \\
(\mathrm{n})\end{array}$} \\
\hline & $M(n)$ & $F(n)$ & $M(n)$ & $F(n)$ & $M(n)$ & $F(n)$ & \\
\hline Piau & 11 & 17 & 17 & 25 & 11 & 6 & 87 \\
\hline Goianá & 36 & 24 & 19 & 26 & 26 & 20 & 151 \\
\hline Chácara & 13 & 15 & 13 & 14 & 15 & 16 & 86 \\
\hline Belmiro Braga & 10 & 30 & 14 & 21 & 5 & 21 & 101 \\
\hline Silveirânia & 19 & 20 & 17 & 19 & 19 & 20 & 114 \\
\hline Coronel Pacheco & 29 & 24 & 21 & 17 & 20 & 14 & 125 \\
\hline Pequeri & 24 & 30 & 12 & 10 & 9 & 9 & 94 \\
\hline Aracitaba & 14 & 21 & 13 & 23 & 8 & 11 & 90 \\
\hline Tabuleiro & 29 & 33 & 22 & 31 & 18 & 34 & 167 \\
\hline Média (士 DP) & \multicolumn{2}{|c|}{$44,33 \pm 13,20$} & \multicolumn{2}{|c|}{$37,11 \pm 9,19$} & \multicolumn{2}{|c|}{$31,33 \pm 12,60$} & \\
\hline Total & \multicolumn{2}{|c|}{399} & \multicolumn{2}{|c|}{334} & \multicolumn{2}{|c|}{282} & 1.015 \\
\hline
\end{tabular}

Fonte: Secretaria das próprias escolas de cada cidade (2009)

a: Média de alunos em cada série do ensino médio.

M: Masculino; F: Feminino; n: número de indivíduos; DP: desvio-padrão.

Os municípios selecionados foram: Goianá (151 alunos), Tabuleiro (167 alunos), Belmiro Braga (101 alunos) e Pequeri (94 alunos). Para atender ao requisito do tamanho amostral mínino calculado, optou-se por realizar um senso com os alunos das quatro escolas, totalizando 513 alunos. Foram excluídos aqueles que apenas estudavam na cidade e aqueles que faziam uso de remédio controlado. Por fim, a pesquisa foi realizada com 413 alunos, com exclusão de 100 indivíduos, mas ainda com o tamanho superior ao tamanho mínimo calculado.

\section{Instrumentos e procedimentos}

Esta pesquisa foi realizada com a autorização da direção de cada escola selecionada, sendo o projeto aprovado pelo Comitê de Ética de Pesquisa (CEP) com seres humanos da UFJF, no dia 19 de fevereiro de 2009, com número do protocolo 1612.302.2008. Os procedimentos de coleta de dados foram realizados nas próprias escolas, sendo os questionários respondidos em sala de aula e as medidas antropométricas, realizadas em uma sala adequada oferecida pela escola, para que a avaliação pudesse ocorrer de forma individual, mantendo, assim, a privacidade de cada participante. O jovem deveria aceitar participar voluntariamente da pesquisa, trazendo assinado pelo responsável o Termo de Consentimento Livre e Esclarecido (TCLE), estar na faixa etária estabelecida (15 a 21 anos) e ser, além de estudante, morador do município.

Os instrumentos selecionados para a avaliação da insatisfação corporal foram o BSQ $^{14}$ e a Escala Evaluacion de Insatisfacion Corporal para Adolescentes (EEICA) ${ }^{15}$, ambos validados e adaptados para a população adolescente brasileira e com conteúdos diferenciados para a avaliação da insatisfação corporal.

O BSQ é um questionário constituído por 34 questões, de autopreenchimento - na forma de escala Likert de pontos, com 6 opções de respostas (1: nunca a 6: sempre), 4 níveis de insatisfação com a aparência física, seguindo o modelo proposto $^{16}$, de acordo com a pontuação: ausência de insa- tisfação corporal - abaixo de 80 pontos; leve insatisfação - a pontuação entre 80 e 110 pontos; moderada insatisfação - a pontuação entre 110 e 140 pontos; grave insatisfação corporal - pontuação igual ou acima de 140 pontos. A avaliação é subjetiva, com relação à forma e preocupação corporais nas últimas 4 semanas. Já a EEICA traz informações referentes à frequência de comportamentos relacionados ao cuidado corporal, à percepção corporal, à influência familiar e social com a insatisfação corporal, sendo composta por 32 questões de autopreenchimento na forma de escala Likert com 6 opções de resposta (1: nunca a 6: sempre), sendo que quanto maior a pontuação, maior a insatisfação corporal.

As variáveis idade, sexo e estado nutricional foram selecionadas para verificar a possível relação com a insatisfação corporal. A idade e o sexo foram obtidos pela informação preenchida pelos respondentes no cabeçalho dos instrumentos. A categorização do período da adolescência foi adotada segundo a classificação da World Health Organization (WHO) ${ }^{11}$ : período intermediário (PI), com idade entre 15 a 17 anos, e período final (PF), idade entre 17 a 21 anos.

O estado nutricional foi avaliado a partir da classificação do índice de massa corporal (IMC) - relação entre peso (kg)/ estatura $^{2}(\mathrm{~m})$ - tendo como critério os valores dos percentis em relação a idade e sexo, proposto por ${ }^{17}$ : IMC baixo os percentis abaixo de 3; IMC normal os percentis entre 3 e 85; sobrepeso os percentis entre 85 e 97; e, para classificação de obesidade, percentil acima de 97.

Os dados foram coletados entre os meses de outubro e dezembro de 2009, com o mínimo de três etapas em cada escola. No primeiro momento, realizou-se uma visita à escola da cidade para confirmar e esclarecer informações com a direção e com os alunos sobre a realização e participação voluntária da pesquisa e a entrega do TCLE.

No segundo momento, em sala de aula, após a entrega do TCLE devidamente assinado pelos responsáveis, os alu- 
nos responderam individualmente ao BSQ e a um cabeçalho de identificação contendo nome (opcional), sexo, idade e data de nascimento. Com a entrega do questionário, o aluno foi encaminhado para uma sala cedida pela escola para a avaliação do peso e estatura. Para tanto, esse aluno, trajando uniforme para a aula de educação física e descalço, foi direcionado até a balança eletrônica do tipo plataforma, com capacidade para 150 kg e graduação em $100 \mathrm{~g}$ da marca G-Tech ${ }^{\oplus}$. Posicionado no meio da plataforma, duas medidas foram realizadas (19). Posteriormente, o aluno se dirigiu ao estadiômetro fixado, posicionando na posição ereta encostado com o dorso na parede, olhando para frente e os pés juntos dos calcanhares encostados. Duas medidas foram aferidas da estatura e considerada a média desse valor ${ }^{18}$.

No terceiro encontro foi aplicado o segundo instrumento de avaliação da insatisfação corporal (EEICA). Os alunos preencheram o cabeçalho de identificação e, posteriormente, foram orientados a responder individualmente às questões medidas em escala. Nesse dia, também foi permitida a inclusão de alunos que quisessem participar da pesquisa por terem faltado em algum procedimento anterior. Após o procedimento de coleta, os dados foram levados para interpretação e análise no LABESC-FAEFID/UFJF.

\section{Análise estatística}

Para análise estatística, realizou-se uma análise descritiva de cada variável, destacando os valores médios, medianas, valores mínimos, máximos e os desvios-padrão. O teste de Kolmogorov-Smirnov identificou que as variáveis numéricas não apresentaram normalidade, por isso optou-se por realizar testes não paramétricos ${ }^{19}$. A variação da insatisfação corporal entre os adolescentes das cidades pesquisadas e entre os grupos do estado nutricional foi avaliada pelo teste de Kruskal-Wallis e o teste post hoc de Bonferroni (19). Para a comparação das médias de insatisfação entre ambos os sexos e entre os dois grupos relacionados ao período da adolescência, utilizou-se o teste de Mann-Whitney. Análise de correspondência simples e múltipla foi utilizada para verificar a associação dos valores de insatisfação corporal e os grupos de categorização das variáveis independentes. O software estatístico Statistical Package for the Social Sciences (SPSS), versão 17.0, foi usado com nível de significância adotado de 5\% $(a=0,05)$ para as respectivas análises.

\section{RESULTADOS}

A análise descritiva do sexo, idade e estado nutricional pode ser observada na tabela 2. Dos 413 estudantes que participaram da pesquisa, houve mais meninas que meninos, ficando relativamente bem dividido entre as cidades e entre os períodos da adolescência (PI e PF). O IMC médio geral da amostra correspondeu a $22,06 \pm 3,76 \mathrm{~kg} / \mathrm{m}^{2}$, sendo a maioria dos adolescentes classificados com IMC normal.
O valor da pontuação média do BSQ foi de 66,78 \pm 29,63 pontos, sendo significativamente mais alto nas meninas e nos sujeitos com sobrepeso e obesidade (Tabela 3). A classificação de insatisfação corporal do BSQ constatou que 296 adolescentes $(73,6 \%)$ apresentaram-se livres de insatisfação corporal e $106(24,6 \%)$ alunos mostraram níveis de leve a grave insatisfação com sua aparência física. Entre os sexos, verificou-se que $11,5 \%$ das meninas e $3,5 \%$ dos meninos apresentaram classificação de moderada e grave insatisfação corporal. Já entre os grupos de classificação do estado nutricional, os indivíduos com sobrepeso $(25,9 \%)$ e obesidade $(18,9 \%)$ tiveram maior frequência nas classificações moderada e grave insatisfação corporal (BSQ) quando comparados aos jovens com IMC normal (4,7\%). Porém, vale ressaltar que $30,6 \%$ das meninas com IMC normal apresentaram algum nível de insatisfação corporal.

Pela avaliação da EEICA, observou-se uma pontuação média de 17,96 $\pm 11,74$ pontos. A diferença significativa de insatisfação corporal não foi constatada pela EEICA; somente as meninas da cidade de Goianá mostraram-se mais insatisfeitas que os meninos (Tabela 4). Em relação os grupos de classificação do estado nutricional, aqueles com sobrepeso e obesidade foram também mais insatisfeitos que aqueles adolescentes com IMC baixo e normal.

A prevalência de insatisfação corporal apresentou uma variação significativa $(p<0,05)$ entre as quatro cidades pesquisadas, sendo a cidade de Belmiro Braga a com maiores índices de insatisfação corporal. Ainda observando essa variação de insatisfação corporal, apenas entre as meninas das diferentes cidades esse aspecto pode ser constatado (Tabela 3).

Tabela 2. Análise descritiva da frequência de indivíduos em cada cidade e grupos de classificação do estado nutricional em relação ao sexo e período da adolescência. Juiz de Fora, 2011

\begin{tabular}{lccccc}
\hline \multirow{2}{*}{ Amostra } & \multicolumn{2}{c}{ Sexo } & \multicolumn{2}{c}{ Período Adolescência } & $\begin{array}{c}\text { Frequência } \\
\text { total }\end{array}$ \\
\cline { 2 - 6 } & $\mathbf{M}(\%)$ & $\mathbf{F}(\%)$ & $\mathrm{PI}(\%)$ & $\mathrm{PF}(\%)$ & $\mathbf{n}(\%)$ \\
\hline Cidades & & & & & \\
Goianá & $66(50)$ & $66(50)$ & $78(59,1)$ & $54(40,1)$ & $132(32)$ \\
Tabuleiro & $60(45,5)$ & $72(55,5)$ & $58(43,9)$ & $74(56,1)$ & $132(32)$ \\
Belmiro Braga & $26(29,1)$ & a $63(70,8)$ & $46(51,7)$ & $43(48,3)$ & $89(21,5)$ \\
Pequeri & $26(43,3)$ & $34(54,6)$ & $21(35)$ & $39(65)$ & $60(14,5)$ \\
EN & & & & & \\
Baixo IMC & $9(5,1)$ & $5(2,1)$ & $9(4,6)$ & $5(2,4)$ & $14(3,4)$ \\
IMC normal & $130(73)$ & $167(71,7)$ & $142(72,1)$ & $155(75,6)$ & $297(71,9)$ \\
Sobrepeso & $15(8,4)$ & $39(16,6)$ & $25(12,7)$ & $29(14,1)$ & $54(13,1)$ \\
Obesidade & $19(10,7)$ & $18(7,7)$ & $21(10,7)$ & $16(7,8)$ & $37(9)$ \\
Total & $178(43,1)$ & $235(56,9)$ & $203(49,2)$ & $210(50,8)$ & $413(100)$ \\
\hline
\end{tabular}

a Maior diferença entre os sexos.

M: masculino; F: feminino; Pi: período inicial; PF: período final; N: número de indivíduos; EN: estado nutricional; IMC: índice de massa corporal. 
Tabela 3. Valores de insatisfação corporal do BSQ das cidades de pequeno porte pesquisadas e os grupos de classificação do estado nutricional em relação ao sexo e período da adolescência. Juiz de Fora, 2011

\begin{tabular}{|c|c|c|c|c|c|c|c|c|}
\hline \multirow{3}{*}{$\begin{array}{l}\quad \text { Cidades/EN } \\
\text { Cidades }\end{array}$} & \multicolumn{8}{|c|}{ Pontuação BSQ } \\
\hline & \multicolumn{3}{|c|}{ Sexo } & \multicolumn{3}{|c|}{ Período da adolescência } & \multicolumn{2}{|c|}{ Escore total } \\
\hline & $M \pm D P$ & $F \pm D P$ & pa & $\mathrm{Pl} \pm \mathrm{DP}$ & $P F \pm D P$ & $\mathrm{pb}$ & & pc \\
\hline Goianá & $55,78 \pm 22,35$ & $81,17 \pm 31,04$ & $0,0001^{d}$ & $71,10 \pm 33,79$ & $64,68 \pm 23,05$ & 0,69 & $68,37 \pm 29,7^{\sharp \#}$ & $0,007^{e}$ \\
\hline Tabuleiro & $54,73 \pm 22,32$ & $66,63 \pm 32,51$ & $0,006^{d}$ & $59,48 \pm 25,58$ & $62,59 \pm 31,03$ & 0,65 & $61,22 \pm 28,8$ & \\
\hline Bel. Braga & $63,03 \pm 30,33$ & $74,36 \pm 26,76$ & $0,023^{\mathrm{d}}$ & $70,71 \pm 29,1$ & $71,20 \pm 27,38$ & 0,68 & $70,94 \pm 28,2^{\# \#}$ & \\
\hline Pequeri & $62,43 \pm 29,4$ & $74,88 \pm 33,1$ & 0,177 & $73,85 \pm 31,4$ & $67,70 \pm 32,56$ & 0,49 & $69,85 \pm 32,0$ & \\
\hline \multicolumn{9}{|l|}{ EN } \\
\hline Baixo IMC & $57,8 \pm 16,5$ & $58,6 \pm 22,0$ & 0,947 & $58,4 \pm 20,7$ & $57,60 \pm 14,31$ & 0,84 & $58,1 \pm 18,14$ & $0,000^{f}$ \\
\hline Normal IMC & $52,14 \pm 18,2$ & $69,41 \pm 29,5$ & 0,0001 & $62,6 \pm 28,9$ & $61,16 \pm 24,34$ & 0,52 & $61,8 \pm 26,6$ & \\
\hline Sobrepeso & $88,06 \pm 40,0$ & $89,43 \pm 32,1$ & 0,685 & $87,92 \pm 29,8$ & $90,03 \pm 37,91$ & 0,97 & $89,0 \pm 34,1$ & \\
\hline Obesidade & $68,84 \pm 30,7$ & $85,94 \pm 32,1$ & 0,045 & $83,66 \pm 31,6$ & $68,62 \pm 31,47$ & 0,07 & $77,1 \pm 32,1$ & \\
\hline Total & $57,39 \pm 29,7$ & $73,88 \pm 31,0$ & 0,0001 & $67,87 \pm 30,6$ & $65,74 \pm 28,70$ & 0,81 & $66,78 \pm 29,6$ & \\
\hline
\end{tabular}

EN: estado nutricional; M: masculino; F: feminino; DP: desvio-padrão; PI: período intermediário; PF: período final; IMC: índice de massa corporal.

a: Valor do teste de Mann-Whitney entre os sexos; b: valor do teste de Mann-Whitney entre os períodos da adolescência; $c$ : valor do teste de Kruskall-Wallis entre as cidades e entre os grupos do estado nutricional; d: diferença significativa entre os sexos $-p<0,05$; e: variação significativa de insatisfação corporal entre as cidades $-p<0,0164$; f: variação significativa entre as classificações do $E N-p<0,016$, 非: diferença significativa ( $p<0,016$ ) entre as cidades de Belmiro Braga e Goianá.

Tabela 4. Valores de insatisfação corporal da Escala Evaluacion de Insatisfacíon Corporal para Adolescentes (EEICA) das cidades de pequeno porte pesquisadas e os grupos de classificação do estado nutricional em relação ao sexo e período da adolescência. Juiz de Fora, 2011

\begin{tabular}{|c|c|c|c|c|c|c|c|c|}
\hline \multirow{3}{*}{$\begin{array}{l}\text { Cidades/EN } \\
\text { Cidades }\end{array}$} & \multicolumn{8}{|c|}{ Pontuação BSQ } \\
\hline & \multicolumn{3}{|c|}{ Sexo } & \multicolumn{3}{|c|}{ Período da adolescência } & \multicolumn{2}{|c|}{ Escore total } \\
\hline & $M \pm D P$ & $F \pm D P$ & $\mathrm{pa}$ & $P I \pm D P$ & $P F \pm D P$ & $\mathrm{pb}^{\mathrm{b}}$ & & $p$ \\
\hline Goianá & $14,60 \pm 6,8$ & $21,69 \pm 14,0$ & $0,006^{e}$ & $18,78 \pm 13,1$ & $17,24 \pm 8,9$ & 0,69 & $18,15 \pm 11,5$ & 0,996 \\
\hline Tabuleiro & $16,15 \pm 7,5$ & $18,52 \pm 12,7$ & 0,945 & $15,93 \pm 9,9$ & $18,63 \pm 11,2$ & 0,65 & $17,44 \pm 10,7$ & \\
\hline Bel. Braga & $17,80 \pm 14,6$ & $17,67 \pm 10,5$ & 0,657 & $16,46 \pm 10,2$ & $18,97 \pm 13,4$ & 0,68 & $17,71 \pm 11,9$ & \\
\hline Pequeri & $19,13 \pm 11,4$ & $19,05 \pm 15,8$ & 0,271 & $18,57 \pm 10,9$ & $19,35 \pm 15,7$ & 0,49 & $19,08 \pm 14,1$ & \\
\hline \multicolumn{9}{|l|}{ EN } \\
\hline Baixo IMC & $12,0 \pm 5,2$ & $13,40 \pm 12,3$ & 0,639 & $12,22 \pm 9,8$ & $13,0 \pm 3,53$ & 0,84 & $12,5 \pm 8,01$ & $0,000^{d}$ \\
\hline Normal IMC & $14,65 \pm 5,9$ & $17,85 \pm 12,5$ & 0,467 & $15,88 \pm 10,1$ & $16,96 \pm 10,4$ & 0,52 & $16,4 \pm 10,27$ & \\
\hline Sobrepeso & $25,53 \pm 20,2$ & $24,02 \pm 13,7$ & 0,702 & $21,66 \pm 11,5$ & $26,96 \pm 18,5$ & 0,97 & $24,4 \pm 15,7^{e}$ & \\
\hline Obesidade & $21,94 \pm 11,2$ & $24,94 \pm 15,1$ & 0,456 & $25,20 \pm 15,4$ & $21,25 \pm 9,8$ & 0,07 & $23,4 \pm 13,2^{e}$ & \\
\hline Total & $16,18 \pm 9,3$ & $19,31 \pm 13,1$ & 0,153 & $17,43 \pm 11,4$ & $18,46 \pm 12,0$ & 0,81 & $17,96 \pm 11,7$ & \\
\hline
\end{tabular}

EN: estado nutricional; M: masculino; F: feminino; DP: desvio-padrão; Pl: período intermediário; PF: período final; IMC: índice de massa corporal.

a: valor do teste de Mann-Whitney entre os sexos; b: valor do teste de Mann-Whitney entre os períodos da adolescência; c: valor do teste de Kruskall-Wallis entre as cidades e entre os grupos do estado nutricional; d: diferença significativa entre os sexos em Goianá - $p<0,05$; e: variação significativa entre as classificaçōes do $\mathrm{EN}-p<0,016$.

Não houve diferença significativa de insatisfação corporal entre o PI e PF da adolescência pela análise de ambos os instrumentos, porém, vale ressaltar que os meninos no PF mostraram-se significativamente $(p<0,05)$ mais insatisfeitos com sua imagem corporal que os meninos do PI pela avaliação da EEICA (Tabela 4).

A figura 1 apresentou um conjunto de gráficos de análise de correspondência simples e múltipla, mostrando primeiramente na figura 1A que os grupos de classificação de insatisfação corporal do BSQ tiveram correspondência com a pontuação da EEICA, ou seja, aqueles indivíduos com maior pontuação na EEICA (> 21 pontos) corresponderam às classificações grave e moderada insatisfação do BSQ. As figuras $1 \mathrm{~B}$ e $1 \mathrm{C}$ se referem à classificação da insatisfação corporal entre as diferentes cidades selecionadas; pela figura 1B ficou evidente a correspondência entre a cidade de Belmiro Braga com os grupos de classificação leve e grave insatisfação, respectivamente. Por fim, na figura 1D pode-se observar que $\mathrm{o}$ sexo feminino e a classificação sobrepeso e obesidade (ambos os sexos) do estado nutricional foram as variáveis independentes que tiveram relação com as maiores incidências de insatisfação corporal. 


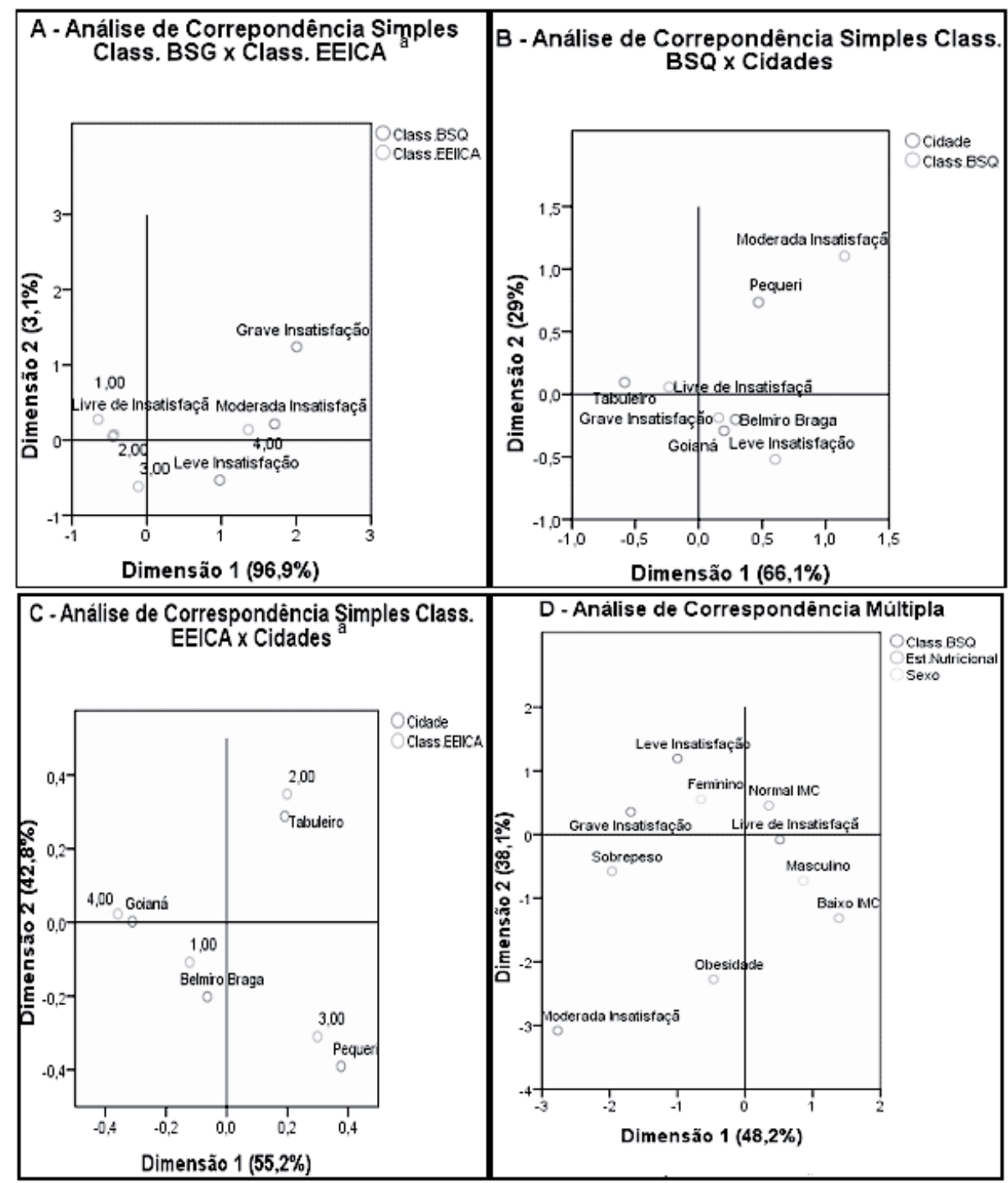

a Classificação da EEICA baseada nos quartis: 1,00: 0 a 11 pontos; 2,00: 12 a 15 pontos; 3,00: 16 a 21 pontos; 4,00: > 21 pontos.

Figura 1. Análise de correspondência entre as cidades de pequeno porte da Zona da Mata mineira, variáveis independentes e grupos de classificação da insatisfação corporal do Body Shape Questionnaire (BSC) e da Escala Evaluacíon de Insatisfacíon Corporal para Adolescentes (EEICA). Juiz de Fora - MG, 2011. (A) Grupos de insatisfação corporal do BSQ e grupos de insatisfação baseados nos quartis da EEICA; (B e C): análise de correspondência simples entre as cidades e grupos de classificação do BSQ e grupos dos quartis da EEICA; (D): Análise de correspondência múltipla com os grupos de estado nutricional e sexo.

\section{DISCUSSÃO}

Observamos que foi baixa a prevalência da insatisfação corporal de adolescentes nas cidades pesquisadas. No entanto, embora reduzido, alguns jovens, principalmente as meninas e aqueles indivíduos com sobrepeso e obesidade, manifestaram altos índices de rejeição com a própria aparência física (Figura 1D). E, ainda, entre as cidades com características sociodemográficas semelhantes registrou-se uma variação significativa dos índices de insatisfação corporal.

Em países desenvolvidos, tanto em adolescentes quanto em adultos, a insatisfação com a imagem corporal atinge entre 50\% a 70\% dessa população². Estudos no Brasil mostraram que a insatisfação corporal entre adolescentes e crianças varia de $64 \%$ a $82 \%{ }^{20}$. No estudo de Vilela et al. ${ }^{21}, 59 \%$ das crianças e adolescentes do interior de Minas Gerais estavam infelizes com sua a aparência. Nesta pesquisa os resultados mostraram que a prevalência de insatisfação corporal foi de 26,4\% (BSQ) nos jovens residentes em cidades com até cinco mil habitantes, sendo mais baixa que os estudos correspondentes publicados atualmente.

A idade não apresentou relação com a insatisfação corporal, pois não houve diferença significativa e também não foi observada associação com os valores de insatisfação cor- 
poral entre adolescentes do Pl e PF. Esses resultados estão de acordo com a pesquisa de Cafri et al. ${ }^{10}$, em que a idade não teve significância estatística enquanto moderador da relação entre internalização e sensibilização com a imagem corporal. Porém, vale ressaltar que os meninos no PF com maior IMC foram significativamente mais insatisfeitos que os meninos do PI com menor IMC. Algumas pesquisas atuais mostram que em idade pós-menarca as meninas apresentam o desejo de diminuir seu peso corporal ${ }^{8}$.

Em relação ao sexo, verificou-se que $35,4 \%$ das meninas apresentaram algum nível de insatisfação, contra $14,4 \%$ dos meninos. Nos países desenvolvidos, aproximadamente de $40 \%$ a $70 \%$ das jovens do sexo feminino estão insatisfeitas com seus corpos, sendo que a metade delas aspira um corpo mais delgado ${ }^{22}$. Pela perspectiva sociocultural é possível compreender essa diferença, pois meninos e meninas recebem diferentes estímulos culturais e sociais, os quais podem influenciar diretamente nos valores e comportamentos individuais em relação à aparência física²3.

Por outro lado, pesquisadores destacam que os adolescentes se preocupam com o peso e com a aparência corpo$\mathrm{ral}$, independentemente do sexo $\mathrm{O}^{2,22}$. Essa constatação pode justificar as diferenças significativas de insatisfação corporal que ocorreram entre as cidades e entre os sexos terem sido observadas apenas pela avaliação do BSQ. Esse instrumento traz em seu conteúdo questionamentos relacionados ao desejo subjetivo com a forma física.

Nessa pesquisa, o estado nutricional foi o fator mais determinante na análise da incidência de insatisfação corporal nos escolares das cidades pequenas pesquisadas. Uma variação da insatisfação corporal entre os grupos de classificação do estado nutricional em cada sexo foi observada em ambos os sexos (Tabelas 3 e 4). É importante ressaltar que o adolescente pode ganhar cerca de $20 \%$ da altura do adulto e $50 \%$ da massa corporal ${ }^{24,25}$. Além do aumento natural do peso que acontece, o crescimento acelerado do número de jovens com sobrepeso tem sido observado pelas pesquisas no Brasil26-28. Outros estudos destacam os distúrbios na esfera emocional que o excesso de peso pode causar em adultos e principalmente entre os adolescentes ${ }^{24,29}$. As meninas jovens com sobrepeso têm 11 vezes mais chance de apresentar insatisfação corporal do que meninas eutróficas ${ }^{20}$.

Algumas observações importantes merecem ser destacadas na figura 1. Na figura 1A, houve correspondência na avaliação da insatisfação corporal entre o BSQ e a EEICA, mostrando que ambos avaliaram o mesmo aspecto da dimensão atitudinal da imagem corporal. As figuras 1B e 1C mostraram a correspondência dos grupos de classificação de insatisfação corporal entre as cidades, principalmente entre os grupos de classificação do BSQ. O gráfico de correspondência múltipla (Figura 1D) demonstrou que as meninas (mesmo as eutróficas) e aqueles (meninos e meninas) com sobrepeso e obesidade tiveram relação com insatisfação corporal. Nesse gráfico, a dimensão 1 (horizontal) explicou 48,2\% de variância entre os grupos de variáveis e a dimensão 2 (vertical), 38,1\% da variância, respectivamente. Notou-se, na dimensão 1, que, abaixo da origem (média), encontram-se os grupos das categorias das variáveis que tiveram associação significativa com os resultados excessivos de insatisfação corporal: grave insatisfação, sobrepeso, obesidade e sexo feminino. Já acima da origem, encontram-se os grupos das categorias das variáveis que apresentaram associação significativa com a ausência ou os baixos índices de insatisfação corporal: livre de insatisfação, baixo IMC e sexo masculino.

A partir das análises realizadas, podemos compreender melhor o quanto a aparência física é um importante atributo na adolescência, determinada social e culturalmente ${ }^{23,27}$ e, talvez, por isso, possamos sugerir que a insatisfação corporal é uma realidade no adolescente e parece não estar atrelada à região demográfica específica em que se vive, mas sim a um contexto sociocultural mais abrangente. Esse fato pode justificar a diferença significativa de insatisfação corporal encontrada entre as cidades investigadas, observando a correção de Bonferroni $(p=0,016)$, visto que a maior prevalência de insatisfação corporal em Belmiro Braga pode ter ocorrido pelo fato de esse município ter apresentado maior frequência de meninas, as quais foram significativamente mais insatisfeitas que os meninos (Tabelas 2, 3 e Figura 1B).

A insatisfação com o corpo já afeta grande proporção dos pré-adolescentes das pequenas cidades do interior do Brasil, embora os menores índices tenham sido encontrados em um grande centro urbano da mesma Região Sul do Brasili ${ }^{30}$. Outros encontraram semelhança nos níveis de insatisfação corporal entre adolescentes da área urbana e rural ${ }^{6}$.

Todavia, o estudo apresentou algumas limitações metodológicas que merecem ser consideradas: o desenho do tipo transversal não permitiu que fosse realizada inferência de causalidade; isso significa que não temos como avaliar o grau de intensidade e a direção das associações encontradas entre a insatisfação corporal, sexo e estado nutricional. Na realidade, essa é uma limitação do estudo transversal, por apresentar um retrato instantâneo do contexto pesquisado. Outro aspecto a ser citado diz respeito ao fato de não ter sido incluído protocolo de questões referente aos aspectos socioculturais. Embora essas informações fossem pertinentes e esclarecedoras, não contemplavam o objetivo da presente pesquisa. Mesmo assim, acreditamos que o trabalho forneceu informações relevantes acerca da insatisfação corporal de adolescentes que vivem em municípios pequenos.

\section{CONCLUSÃO}

Concluiu-se pelas análises realizadas que os adolescentes das cidades de pequeno porte da Zona da Mineira apresentaram baixa prevalência de insatisfação corporal. Também 
foi constatado que entre as cidades pesquisadas houve variação significativa de insatisfação corporal, bem como a influência do sexo e, principalmente, do estado nutricional. Mais estudos epidemiológicos de base escolar são necessários para verificar como outras variáveis podem influenciar na formação negativa da imagem corporal. A escola seria um espaço privilegiado para conscientizar os jovens e seus familiares sobre a natureza das mudanças que ocorrem durante a adolescência, bem como para aprender a conviver de forma saudável com a nova aparência física que está sendo formada durante o desenvolvimento humano.

\section{AGRADECIMENTOS}

A todas as escolas pela disponibilidade e pelo incentivo para que os alunos participassem desta pesquisa.

\section{CONFLITOS DE INTERESSE}

Os autores declararam não haver conflito de interesse.

\section{REFERÊNCIAS}

1. Slade PD. What is body image? Behav Res Ther. 1994;32(5):497-502.

2. McCabe MP, Ricciardelli LA. A longitudinal study of pubertal timing and extreme body change behaviors among adolescent boys and girls. Adolescence. 2004;39(153):145-66.

3. Papalia DE. Desenvolvimento humano. 8. ed. São Paulo: Artmed; 2008.

4. Campagna VN, Souza ASL. Corpo e imagem corporal no início da adolescência feminina. Bol Psicol. 2006;56:9-35.

5. Alvarenga MS, Philippi ST, Lourenço BH, Sato PM, Scagliusi FB. Insatisfação com a imagem corporal em universitárias brasileiras. J Bras Psiquiatr. 2010;59(1):44-51.

6. Petroski EL, Pelegrini A, Glaner MF. Insatisfação corporal em adolescentes rurais e urbanos. Motricidade. 2009;5(4):13-25.

7. Fidélix YL, Silva DAS, Pelegrini A, Silva AS, Petroski EL. Insatisfação com a imagem corporal em adolescentes de uma cidade de pequeno porte: associação com sexo, idade e zona de domicílio. Rev Bras Cineantropom Desempenho Hum. 2011;13(3):202-7.

8. Scherer FC, Martins CR, Pelegrini A, Matheus SC, Petroski EL. Imagem corporal em adolescentes: associação com a maturação sexual e sintomas de transtornos alimentares. J Bras Psiquiatr. 2010;59(3):198-202.

9. Thompson JK. The (mis)measurement of body image: ten strategies to improve assessment for applied and research purposes. Body Image. 2004;1(7):7-14.
10. Cafri G, Yamamiya Y, Brannick M, Thompson JK. The influence of Sociocultural Factors on Body Image: a meta-analysis. Clin Psychol: Scienc Practic. 2005;12(4):421-33.

11. World Health Organization (WHO). Nutrition in adolescence: issues and challenges for the health sector: issues in adolescent health and development. Geneva: World Health Organization; 2005.

12. Instituto de Pesquisa Econômica Aplicada/Unicamp.IE.Nesur/IBGE (Org.). Série Caracterização e Tendências da Rede Urbana do Brasil. Brasília: IPEA; 2001.

13. Instituto Brasileiro de Geografia e Estatística (IBGE) [Internet]. Censo 2000: Cidades. Rio de Janeiro; 2008 [citado 2019 Mai. 5]. Disponível em: <http://www.ibge.com.br/cidadesat

14. Conti MA, Cordás TA, Latorre MRO. A study of the validity and reability of the Brasilian verson of the Body Shape Questionnaire (BSQ) among adolescents. Rev Bras Saúde Matern Infant. 2009;9(3):331-8.

15. Conti MA, Slater B, Latorre MRO. Validação e reprodutibilidade da Escala de Evaluación de Insatisfación Corporal para Adolescentes. Rev Saúde Pública. 2009;43(3):515-24.

16. Cordás TA, Castilho S. Body image on the eating disorders - evaluation instruments: "Body Shape Questionnaire". Psiquiatr Biol. 1994;2:17-21.

17. De Onis M, Onyango AW, Borghi E, Siyam A, Nishida C, Siekmann J. Development of a WHO growth reference for school-aged children and adolescents. Bull World Health Organ. 2007;85(9):660-7.

18. Gordon CC, Chumlea WC, Roche AF. Stature, recumbent length, and weight. In: Lohman $T G$, Roche AF, Martorell R. Anthropometric standardization reference manual. Champaing: Human Kinetics Books; 1988.

19. Sheskin DJ. Handbook of parametric and non parametric statistical procedures. 3. ed. Boca Raton: Chapman Works; 2004.

20. Pelegrini A, Petroski EL. The association between body dissatisfaction and nutritional status in adolescents. Hum Mov. 2010;11(5):51-7.

21. Vilela J, Lamounier JA, Filho MAD, Neto JRB, Horta GM. Transtornos alimentares em escolares. J Pediatr. 2004;80:49-54.

22. Van den Berg P, Paxton SJ, Keery H, Wall M, Neumark-Sztainer D. Body dissatisfaction and body comparison with media images in males and females. Body Image. 2007;4:257-68.

23. White J, Halliwell E. Examination of a sociocultural model of excessive exercise among male and female adolescents. Body Image. 2010;7(3):227-33.

24. Wilkosz ME, Chen JL, Kenddey C, Rankin S. Body dissatisfaction in California adolescents. J Am Acad Nurse Pract. 2011;23(2):101-9.

25. Burbano JC, Fornasini M, Acosta M. Riesgo de sobrepeso en colegialas de 12 a 19 años en una región semiurbana del Ecuador. Rev Panam Salud Publica. 2003;13(5):277-84.

26. Branco LM, Hilário MOE, Cintra IP. Percepção e satisfação corporal em adolescentes e a relação com seu estado nutricional. Rev Psiq Clin. 2006;33:292-6.

27. Araújo CL, Dumith SC, Menezes AMB, Hallal PC. Peso medido, peso percebido e fatores associados em adolescentes. Rev Panam Salud Publica. 2010;27(5):360-7.

28. Conti MA, Frutuoso MFP, Gambardella AMD. Excesso de peso e insatisfação corporal em adolescentes. Rev Nutr. 2005;18:491-7.

29. Bosi MLM, Uchimura KY, Luiz RR. Comportamento alimentar e imagem corporal entre estudantes de Psicologia. J Bras Psiquiatr. 2009;58:150-5.

30. Triches RM, Giugliani ERJ. Insatisfação corporal em escolares de dois municípios da região Sul do Brasil. Rev Nutr. 2007;20:119-28. 